\title{
Plantas medicinais utilizadas no tratamento de problemas bucais no estado da Paraíba, Brasil: uma revisão de literatura
}

Medicinal plants used in the treatment of problems state of Paraíba, Brazil: a literature review Plantas medicinales utilizadas en el tratamiento de problemas estado de Paraíba, Brasil: una revisión de literatura

Daiane Sousa MEDEIROS ${ }^{1}$

Marco Antonio Lavorato de ALMEIDA ${ }^{2}$

Rebecca Rhuanny Tolentino LIMEIRA ${ }^{3}$

Candice Regadas Gondim SANTIAGO

Maria Rejane Cruz ARAÚJO5

José Klidenberg de OLIVEIRA-JÚNIOR ${ }^{6}$

Edeltrudes de Oliveira LIMA $^{7}$

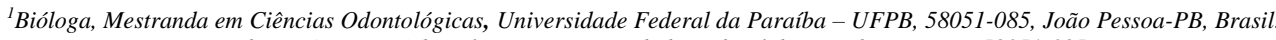

${ }^{2}$ Cirurgião-Dentista, Mestrando em Ciências Odontológicas, Universidade Federal da Paraíba - UFPB, 58051-085, João Pessoa-PB, Brasil.

${ }^{3}$ Fonoaudióloga, Mestranda em Ciências Odontológicas, Universidade Federal da Paraíba - UFPB, 58051-085, João Pessoa-PB, Brasil.

${ }^{4}$ Cirurgiã-Dentista, Mestre em Ciências Odontológicas, Universidade Federal da Paraíba - UFPB, 58051-085, João Pessoa-PB, Brasil.

${ }^{5}$ Cirurgiã-Dentista, Mestranda em Ciências Odontológicas, Universidade Federal da Paraíba-UFPB, 58051-085, João Pessoa-PB, Brasil.

${ }^{6}$ Cirurgião-Dentista, Mestre em Ciências Odontológicas, Universidade Federal da Paraíba-UFPB, 58051-085, João Pessoa-PB, Brasil.
\end{abstract}

${ }^{7}$ Doutora. Programa de Pós-Graduação em Odontologia, Universidade Federal da Paraíba - UFPB, 58051-085, João Pessoa - PB, Brasil

\section{Resumo}

Introdução: O uso de plantas medicinais como agente terapêutico é secularmente manifestado e aplicado em diferentes culturas pelo mundo. O estado da Paraíba, localizado na região nordeste do Brasil, apresenta grande parte de sua extensão territorial recoberta pelo clima semiárido, cuja vegetação do tipo caatinga representa uma importante fonte de biomoléculas ativas à saúde. Objetivo: O objetivo desta pesquisa foi realizar um levantamento na literatura sobre dos conhecimentos etnobotânicos da população paraibana no uso de plantas medicinais no tratamento de afecções orais. Material e Método: Realizou-se uma pesquisa nas bases dedados Scielo e Bireme, durante os meses de outubro a novembro de 2017. Encontrou-se 81 artigos, dos quais 17 foram analisados e 10 foram incluídos nesta pesquisa. Resultados: A população relatou o uso de 65 espécies de plantas medicinais na odontologia, pertencentes a 60 gêneros e 34 famílias distinta, com predomínio da família Fabaceae. As espécies mais relatadas pela população foram Punica granatum, Anacardium occidentale e Plectranthus amboinicus. As principais indicações terapêuticas foram antimicrobiana, anti-inflamatória e analgésica. O chá foi o tipo de preparo mais relatado e as folhas e cascas de caule as estruturas vegetais mais utilizadas no preparo. Conclusão: Conclui-se então que o levantamento etnobotânico é de fundamental importância para ampliar o conhecimento científico acerca do uso de plantas medicinais e subsidiar o desenvolvimento de futuros fármacos.

Descritores: Etnobotânica; Plantas Medicinais; Fitoterapia; Odontologia.

\section{Abstract}

Introduction: The use of medicinal plants as a therapeutic agent is secularly manifested and applied in different cultures around the world. Paraíba State, located in the northeastern region of Brazil, presents a great part of its territorial extension covered by the semi-arid climate, whose vegetation of the caatinga type represents an important source of biomolecules active to health. Objective: The objective of this research was to conduct a survey in the literature on the ethnobotanical knowledge of the population of Paraíba in the use of medicinal plants in the treatment of oral diseases. Material and Methods: A survey was carried out at the databases Scielo and Bireme during the months of October to November 2017. 81 articles were found, of which 17 were analyzed and 10 were included in this study. Results: The population reported the use of 65 species of medicinal plants in dentistry, belonging to 60 genera and 34 distinct families, with a predominance of the Fabaceae family. The species most reported by the population were Punica granatum, Anacardium occidentale and Plectranthus amboinicus. The main therapeutic indications were antimicrobial, anti-inflammatory and analgesic. The tea was the most reported type of preparation and the leaves and bark of stalks the plant structures most used in the preparation. Conclusion: It is concluded that the ethnobotanical survey is of fundamental importance to increase the scientific knowledge about the use of medicinal plants and to subsidize the development of future drugs.

Descriptors: Ethnobotany; Plants, Medicinal; Phytotherapy; Dentistry.

\section{Resumen}

Introducción: El uso de plantas medicinales como agente terapéutico es secularmente manifestado y aplicado en diferentes culturas por el mundo. La región da Paraíba, ubicada en la parte nordeste de Brasil, presenta gran parte de su extensión territorial recubierta por el clima semiárido, cuya vegetación del tipo caatinga representa una importante fuente de biomoléculas activas a la salud. Objetivo: El objetivo de esta investigación fue realizar un levantamiento en la literatura sobre los conocimientos etnobotánicos de la población paraibana en el uso de plantas medicinales en el tratamiento de afecciones orales. Material y Método: Se realizó una investigación en las bases dedicadas Scielo y Bireme, durante los meses de octubre a noviembre de 2017. Se encontraron 81 artículos, de los cuales 17 fueron analizados y 10 fueron incluidos en esta investigación. Resultados: La población relató el uso de 65 especies de plantas medicinales en la odontología, pertenecientes a 60 géneros y 34 familias distintas, con predominio de la familia Fabaceae. Las especies más relatadas por la población fueron Punica granatum, Anacardium occidentale y Plectranthus amboinicus. Las principales indicaciones terapéuticas fueron antimicrobiana, anti-inflamatoria y analgésica. El té fue el tipo de preparación más relatado y las hojas y cáscaras de las estructuras vegetales más utilizadas en la preparación. Conclusión: Se concluye entonces que el levantamiento etnobotánico es de fundamental importancia para ampliar el conocimiento científico acerca del uso de plantas medicinales y subsidiar el desarrollo de futuros fármacos.

Descriptores: Etnobotánica; Plantas Medicinales; Fitoterapia; Odontología.

\section{INTRODUÇÃO}

O uso de plantas medicinais como agente terapêutico é secularmente manifestado e aplicado em diferentes culturas pelo mundo. Estima-se que, atualmente, cerca de $80 \%$ da população mundial utilizam plantas medicinais ou preparados oriundos destas na prevenção e cuidado à saúde ${ }^{1}$.

No Brasil, o uso dos conhecimentos etnobotânicos está diretamente ligado à história da saúde pública do país, uma vez que, no período colonial saberes empíricos provenientes de indígenas, africanos e portugueses, eram utilizados em diferentes tipos de rituais, entre eles o de cura ${ }^{2}$.

Conhecida por ser uma ciência que estuda os conhecimentos tradicionais da população acerca da 
utilização das plantas e das relações diretas existentes entre os seres humanos e os vegetais, a etnobotânica, é uma ciência que pode caracterizar o perfil de uma comunidade bem como o modo como esta vê e a convive com a natureza ${ }^{3,4}$.

A Paraíba, localizada na região nordeste do Brasil, é um estado que apresenta grande parte de sua extensão territorial recoberta pelo clima semiárido, cujo a vegetação do tipo caatinga representa uma importante fonte de biomoléculas ativas à saúde, sendo ainda pouco exploradas ${ }^{5}$. Sua população é detentora de um vasto conhecimento etnobotânico acerca do uso e aplicação de plantas medicinais na prevenção e promoção do cuidado à saúde, conhecimento influenciado pela cultura de comunidades sertaneja, quilombola e indígena existentes no local, e que pode servir como alicerce para construção de importantes conhecimentos científicos na área da saúde ${ }^{6,7}$.

Embora o número de estudos acerca da atividade farmacológica de plantas medicinais na Odontologia seja grande, ainda é escasso o número de trabalhos etnobotânicos nesta área, com a população paraibana. Neste contexto, o presente trabalho tem como objetivo realizar revisão sistematizada acerca dos conhecimentos etnobotânicos da população paraibana no uso de plantas medicinais no tratamento de afecções orais.

\section{MATERIAL E MÉTODO}

Este estudo consistiu de uma revisão de literatura, realizada por técnica documental indireta, nas bases de dados SCIELO (http://www.scielo.org/php/index.php) e BIREME (http://bvsalud.org/), durante os meses de outubro a novembro de 2017.

Foram revisados e analisados artigos científicos que abordasse o conhecimento etnobotânico da população paraibana na odontologia, utilizando como instrumento de coleta de dados questionários préestruturados.

Os seguintes descritores, em português e seus respectivos descritores em inglês foram utilizados: Etnobotânica, Plantas Medicinais, Fitoterapia e Odontologia. Como critérios de inclusão foram considerados artigos publicados, independente do ano, que abordasse o uso de plantas medicinais pela população paraibana, através da utilização de questionários semiestruturados ou estruturados.

Inicialmente foram encontrados 81 artigos, dos quais 17 foram analisados por meio da leitura do título e resumo e 10 foram devidamente selecionados após análise criteriosa do conteúdo na íntegra.

Estudos que não se enquadraram nos critérios de inclusão pré-estabelecidos foram excluídos da pesquisa e duplicatas desconsideradas.

RESULTADOS E DISCUSSÃO

De acordo com as bases de dados avaliadas, foi possível encontrar 10 estudos científicos que abordavam o levantamento etnobotânico de plantas medicinais utilizadas pela população paraibana em diferentes municípios do estado (Tabela 1).

Tabela 1. Estudos publicados e municípios estudados, que relatam o uso etnobotânico de plantas medicinais no Estado da Paraíba

\begin{tabular}{|c|c|}
\hline Estudo/ano & Municípios estudados \\
\hline Brito et al. ${ }^{8}$ (2017) & Tambaba \\
\hline Cordeiro e Félix ${ }^{12}$ (2014) & Serra da Raiz \\
\hline Costa e Marinho9 $^{(2016)}$ & Picuí \\
\hline Lucena et al. ${ }^{13}(2013)$ & Cabaceiras \\
\hline Marinho et al.15 (2011) & São José dos Espinharas \\
\hline Pinheiro et al. ${ }^{17}$ (1996) & Mamanguape \\
\hline Santos et al. ${ }^{16}$ (2009) & João Pessoa \\
\hline Silva et al. ${ }^{11}(2015)$ & Solânea \\
\hline Souza et al..$^{10}(2016)$ & Campina Grande \\
\hline Souza et al.14 (2013) & Campina Grande \\
\hline
\end{tabular}

Os estudos etnobotânicos analisados, apontaram cerca de 65 espécies de plantas medicinais utilizadas na odontologia pela população paraibana, distribuídas alfabeticamente em 60 gêneros e 34 famílias distintas (Tabelas 2 a 7).

Tabela 2. Plantas medicinais, formas de preparo, indicações terapêuticas e uso popular na odontologia, segundo os conhecimentos etnobotânico da população paraibana (AMARYLLIDACEAE a APOCYNACEAE)

\begin{tabular}{|c|c|c|c|c|c|}
\hline \multicolumn{6}{|c|}{ FAMÍLIA BOTÂNICA } \\
\hline Espécie & $\begin{array}{c}\text { Nome } \\
\text { popular }\end{array}$ & $\begin{array}{c}\text { Partes } \\
\text { utilizadas }\end{array}$ & $\begin{array}{c}\text { Formas de } \\
\text { preparo }\end{array}$ & $\begin{array}{c}\text { Efeito } \\
\text { terapêutico }\end{array}$ & $\begin{array}{c}\text { Uso/ } \\
\text { Indicacãao } \\
\text { popular }\end{array}$ \\
\hline \multicolumn{6}{|c|}{ AMARYLLIDACEAE } \\
\hline $\begin{array}{c}\text { Allium } \\
\text { sativum L. }\end{array}$ & Alho & Bulbo & Chá & $\begin{array}{c}\text { Analgésico, } \\
\text { antimicrobiano }\end{array}$ & Dor de dente \\
\hline \multicolumn{6}{|c|}{ AMARANTHACEAE } \\
\hline $\begin{array}{l}\text { Chenopodium } \\
\text { ambrosioides } \\
\text { L. }\end{array}$ & Matruz & Folha & $\begin{array}{l}\text { Lambedor, } \\
\text { decoç̧ão }\end{array}$ & $\begin{array}{c}\text { Anti- } \\
\text { inflamatório }\end{array}$ & Inflamações \\
\hline \multicolumn{6}{|l|}{ ANACADIACEAE } \\
\hline $\begin{array}{l}\text { Myracrodruo } \\
\text { n urundevva } \\
\text { Allemão. }\end{array}$ & Aroeira & $\begin{array}{l}\text { Casca, } \\
\text { entrecasca, } \\
\text { folhas }\end{array}$ & $\begin{array}{l}\text { Decocção, } \\
\text { água, } \\
\text { maceração, } \\
\text { garrafada, } \\
\text { infusão }\end{array}$ & $\begin{array}{c}\text { Anti- } \\
\text { inflamatório, } \\
\text { cicatrizante }\end{array}$ & Inflamações \\
\hline $\begin{array}{l}\text { Schinus } \\
\text { terebinthifoli } \\
\text { us Raddi }\end{array}$ & $\begin{array}{c}\text { Aroeira } \\
\text { vermelha }\end{array}$ & $\begin{array}{l}\text { Casca do } \\
\text { Caule }\end{array}$ & Chá & $\begin{array}{l}\text { Anti- } \\
\text { inflamatório }\end{array}$ & Inflamações \\
\hline $\begin{array}{l}\text { Anacardium } \\
\text { occidentale L. }\end{array}$ & $\begin{array}{c}\text { Cajueiro/c } \\
\text { ajueiro } \\
\text { roxo }\end{array}$ & $\begin{array}{c}\text { Casca, } \\
\text { entrecasca }\end{array}$ & $\begin{array}{c}\text { Garrafada, } \\
\text { água, chá, } \\
\text { infusão, } \\
\text { maceração, } \\
\text { decocção }\end{array}$ & $\begin{array}{c}\text { Anti- } \\
\text { inflamatório, } \\
\text { cicatrizante, } \\
\text { antimicrobiano }\end{array}$ & Inflamações \\
\hline $\begin{array}{c}\text { Spondias } \\
\text { tuberosa Arr. } \\
\text { Cam. }\end{array}$ & Umbu & $\begin{array}{l}\text { Folha, } \\
\text { fruto }\end{array}$ & Infusão & -- & $\begin{array}{l}\text { Afecções de } \\
\text { garganta }\end{array}$ \\
\hline \multicolumn{6}{|l|}{ ANNONACEAE } \\
\hline $\begin{array}{c}\text { Annona } \\
\text { muricata L. }\end{array}$ & Graviola & Folhas & $\begin{array}{c}\text { Chá, } \\
\text { garrafada, } \\
\text { tintura }\end{array}$ & $\begin{array}{c}\text { Anti- } \\
\text { inflamatório, } \\
\text { antitumoral }\end{array}$ & Inflamações \\
\hline \multicolumn{6}{|l|}{ APOCYNACEAE } \\
\hline $\begin{array}{c}\text { Rauvolfia } \\
\text { ligustrina } \\
\text { Willd. }\end{array}$ & $\begin{array}{l}\text { Arrebenta } \\
\text { bucho }\end{array}$ & Fruto & $\begin{array}{l}\text { Látex in } \\
\text { natura }\end{array}$ & Analgésico & Dor de dente \\
\hline
\end{tabular}

Tabela 3. Plantas medicinais, formas de preparo, indicações terapêuticas e uso popular na odontologia, segundo os conhecimentos etnobotânico da população paraibana (BRASSICACEAE a BORAGINACEAE)

\begin{tabular}{|c|c|c|c|c|c|}
\hline \multicolumn{6}{|c|}{ FAMÍLIA BOTÂNICA } \\
\hline Espécie & $\begin{array}{c}\text { Nome } \\
\text { popular }\end{array}$ & $\begin{array}{c}\begin{array}{c}\text { Partes } \\
\text { utilizadas }\end{array} \\
\end{array}$ & $\begin{array}{c}\begin{array}{c}\text { Formas de } \\
\text { preparo }\end{array} \\
\end{array}$ & $\begin{array}{c}\text { Efeito } \\
\text { terapêutico }\end{array}$ & $\begin{array}{c}\text { Uso/ } \\
\text { Indicação } \\
\text { popular }\end{array}$ \\
\hline \multicolumn{6}{|l|}{ BRASSICACEAE } \\
\hline $\begin{array}{l}\text { Nasturtium } \\
\text { officinale W. } \\
\text { T. Ailton }\end{array}$ & $\begin{array}{l}\text { Agrião de } \\
\text { remédio }\end{array}$ & Semente & $\begin{array}{l}\text { Chá, } \\
\text { lambedor }\end{array}$ & Analgésico & $\begin{array}{l}\text { Dor de } \\
\text { garganta }\end{array}$ \\
\hline \multicolumn{6}{|l|}{ BIGNONIACEAE } \\
\hline $\begin{array}{l}\text { Handroanthus } \\
\text { impetiginosus } \\
\text { (Mart. ex DC.) } \\
\text { Mattos. }\end{array}$ & $\begin{array}{l}\text { Pau d'arco } \\
\text { roxo }\end{array}$ & Casca & Decocção & $\begin{array}{c}\text { Anti- } \\
\text { inflamatório }\end{array}$ & Inflamações \\
\hline $\begin{array}{l}\text { Handroanthu } \\
\text { s serratifolius } \\
\text { (Vahl.) S. O. } \\
\text { Grose. }\end{array}$ & $\begin{array}{l}\text { Pau d'arco } \\
\text { amarelo }\end{array}$ & Casca & Decocção & $\begin{array}{c}\text { Anti- } \\
\text { inflamatório }\end{array}$ & Inflamações \\
\hline $\begin{array}{c}\text { Jacaranda } \\
\text { jasminoides } \\
\text { (Thunb.) } \\
\text { Sandwith }\end{array}$ & Caroba & $\begin{array}{l}\text { Casca, } \\
\text { folha }\end{array}$ & Decocção & $\begin{array}{c}\text { Anti- } \\
\text { inflamatório }\end{array}$ & Inflamações \\
\hline $\begin{array}{c}\text { Tabebuia } \\
\text { impetiginosa } \\
\text { (Mart. Ex.DC. } \\
\text { Standl) }\end{array}$ & Ipê roxo & Casca & $\begin{array}{l}\text { Chá, } \\
\text { garrafada }\end{array}$ & $\begin{array}{c}\text { Anti- } \\
\text { inflamatório }\end{array}$ & Inflamações \\
\hline $\begin{array}{c}\text { Tabebuia } \\
\text { avellanedae } \\
\text { Lor. ex } \\
\text { Griseb. }\end{array}$ & Ipê roxo & Casca & $\begin{array}{l}\text { Infusão, } \\
\text { maceração, } \\
\text { decocção }\end{array}$ & $\begin{array}{c}\text { Anti- } \\
\text { inflamatório, } \\
\text { antimicrobian } \\
\text { o, antitumoral } \\
\end{array}$ & Gengivite \\
\hline \multirow{3}{*}{$\begin{array}{l}\text { BORAGINACEAE } \\
\text { Symphytum } \\
\text { officinale L. } \\
\text { Heliotropium } \\
\text { indicum DC. }\end{array}$} & & & & & \\
\hline & Confrei & Folha & Lambedor & $\begin{array}{c}\text { Anti- } \\
\text { inflamatório }\end{array}$ & Inflamações \\
\hline & Fedegoso & Raiz & $\begin{array}{c}\text { Xarope, } \\
\text { infusão, } \\
\text { maceraçãoo }\end{array}$ & $\begin{array}{c}\text { Anti- } \\
\text { inflamatório }\end{array}$ & Inflamações \\
\hline
\end{tabular}


Tabela 4. Plantas medicinais, formas de preparo, indicações terapêuticas e uso popular na odontologia, segundo os conhecimentos etnobotânico da população paraibana (CACTACEAE a CUCURBITACEAE)

\begin{tabular}{|c|c|c|c|c|c|}
\hline \multicolumn{6}{|c|}{ FAMÍLIA BOTÂNICA } \\
\hline Espécie & $\begin{array}{c}\text { Nome } \\
\text { popular }\end{array}$ & $\begin{array}{c}\text { Partes } \\
\text { utilizadas }\end{array}$ & $\begin{array}{c}\text { Formas de } \\
\text { preparo }\end{array}$ & $\begin{array}{c}\text { Efeito } \\
\text { terapêutico }\end{array}$ & $\begin{array}{c}\text { Uso/ } \\
\text { Indicação } \\
\text { popular }\end{array}$ \\
\hline CACTACEAE & & & & & \\
\hline $\begin{array}{c}\text { Cereus } \\
\text { jamacaru } \mathrm{DC}\end{array}$ & $\begin{array}{c}\text { Mandacar } \\
\mathrm{u}\end{array}$ & Medula & Xarope & $\begin{array}{c}\text { Anti- } \\
\text { inflamatório }\end{array}$ & Inflamações \\
\hline CELASTRACEAI & & & & & \\
\hline $\begin{array}{c}\text { Maytenus } \\
\text { rigida Mart. }\end{array}$ & Bom nome & $\begin{array}{l}\text { Casca, } \\
\text { folhas }\end{array}$ & $\begin{array}{c}\text { Infusão, } \\
\text { maceraçãoo }\end{array}$ & $\begin{array}{c}\text { Anti- } \\
\text { inflamatório }\end{array}$ & Inflamações \\
\hline CLEOMACEAE & & & & & \\
\hline $\begin{array}{c}\text { Tarenaya } \\
\text { spinosa } \\
\text { (Jacq.) Raf. }\end{array}$ & Mussambê & Flor, raiz & $\begin{array}{c}\text { Chá, } \\
\text { lambedor }\end{array}$ & $\begin{array}{c}\text { Anti- } \\
\text { inflamatório }\end{array}$ & Inflamações \\
\hline CAPPARACEAE & & & & & \\
\hline $\begin{array}{c}\text { Cleome } \\
\text { spinosa Jacq. }\end{array}$ & Mussambê & $\begin{array}{l}\text { Flor, raiz, } \\
\text { planta } \\
\text { inteira } \\
\end{array}$ & $\begin{array}{l}\text { Infusão, } \\
\text { xarope }\end{array}$ & $\begin{array}{c}\text { Anti- } \\
\text { inflamatório }\end{array}$ & Inflamações \\
\hline CRASSULACEAI & & & & & \\
\hline $\begin{array}{l}\text { Bryophyllum } \\
\text { pinnatum } \\
\text { (Lam.) Oken }\end{array}$ & Corama & Folha & Lambedor & $\begin{array}{c}\text { Anti- } \\
\text { inflamatório, } \\
\text { antimicrobian } \\
0\end{array}$ & $\begin{array}{c}\text { Inflamaç̃oes, } \\
\text { infecçồes }\end{array}$ \\
\hline CUCURBITACEA & & & & & \\
\hline $\begin{array}{c}\text { Apodanthera } \\
\text { congestiflora } \\
\text { Cogn. }\end{array}$ & $\begin{array}{c}\text { Cabeça de } \\
\text { nego }\end{array}$ & Raiz & Lambedor & Analgésico & $\begin{array}{c}\text { Dores em } \\
\text { geral }\end{array}$ \\
\hline $\begin{array}{l}\text { Momordica } \\
\text { charantia L. }\end{array}$ & $\begin{array}{l}\text { Melão de } \\
\text { são } \\
\text { Caetano }\end{array}$ & Folhas & Maceração & $\begin{array}{c}\text { Anti- } \\
\text { inflamatório }\end{array}$ & $\begin{array}{c}\text { Inflamações } \\
\text { externas }\end{array}$ \\
\hline
\end{tabular}

Tabela 5. Plantas medicinais, formas de preparo, indicações terapêuticas e uso popular na odontologia, segundo os conhecimentos etnobotânico da população paraibana (EUPHOBIACEAE)

\begin{tabular}{|c|c|c|c|c|c|}
\hline \multicolumn{6}{|c|}{ FAMÍLIA BOTÂNICA } \\
\hline Espécie & $\begin{array}{c}\text { Nome } \\
\text { popular }\end{array}$ & $\begin{array}{c}\text { Partes } \\
\text { utilizadas }\end{array}$ & $\begin{array}{c}\text { Formas de } \\
\text { preparo }\end{array}$ & $\begin{array}{c}\text { Efeito } \\
\text { terapêutico }\end{array}$ & $\begin{array}{c}\text { Uso/ } \\
\begin{array}{c}\text { Indicação } \\
\text { popular }\end{array}\end{array}$ \\
\hline EUPHOBIACEA & & & & & \\
\hline $\begin{array}{l}\text { Cnidoscolus } \\
\text { urens (L.) } \\
\text { Arthur. }\end{array}$ & $\begin{array}{l}\text { Urtiga } \\
\text { branca }\end{array}$ & Raiz & Decocção & $\begin{array}{c}\text { Anti- } \\
\text { inflamatório }\end{array}$ & Inflamação \\
\hline $\begin{array}{c}\text { Croton } \\
\text { nepetifolius } \\
\text { Baill. }\end{array}$ & $\begin{array}{c}\text { Marmeleiro } \\
\text { branco }\end{array}$ & $\begin{array}{l}\text { Casca do } \\
\text { caule }\end{array}$ & Pó & $\begin{array}{c}\text { Anti- } \\
\text { hemorrágico }\end{array}$ & Hemorragias \\
\hline $\begin{array}{l}\text { Jatropha } \\
\text { mollissima } \\
\text { (Pohl) Baill. }\end{array}$ & $\begin{array}{l}\text { Pinhão } \\
\text { bravo }\end{array}$ & Folha & $\begin{array}{l}\text { Látex in } \\
\text { natura }\end{array}$ & Analgésico & Dor de dente \\
\hline $\begin{array}{l}\text { Jatropha } \\
\text { curcas L. }\end{array}$ & Pião bravo & $\begin{array}{l}\text { Látex, } \\
\text { semente }\end{array}$ & $\begin{array}{l}\text { Infusão, } \\
\text { cataplasma }\end{array}$ & $\begin{array}{c}\text { Anti- } \\
\text { hemorrágico }\end{array}$ & Hemorragias \\
\hline $\begin{array}{l}\text { Jatropha } \\
\text { gossypiffolia } \\
\text { L. }\end{array}$ & Pião roxo & $\begin{array}{l}\text { Látex, } \\
\text { semente }\end{array}$ & Infusão & $\begin{array}{c}\text { Anti- } \\
\text { hemorrágico }\end{array}$ & Hemorragias \\
\hline $\begin{array}{l}\text { Croton } \\
\text { campestris } \\
\text { St. Hil. }\end{array}$ & Velame & $\begin{array}{l}\text { Raiz, } \\
\text { planta } \\
\text { inteira }\end{array}$ & $\begin{array}{l}\text { Xarope, } \\
\text { decocção }\end{array}$ & ---- & $\begin{array}{l}\text { Afecções de } \\
\text { garganta }\end{array}$ \\
\hline
\end{tabular}

Tabela 6. Plantas medicinais, formas de preparo, indicações terapêuticas e uso popular na odontologia, segundo os conhecimentos etnobotânico da população paraibana (FABACEAE/LEGUMINOSAE)

\begin{tabular}{|c|c|c|c|c|c|}
\hline \multicolumn{6}{|c|}{ FAMÍLIA BOTÂNICA } \\
\hline Espécie & $\begin{array}{c}\begin{array}{c}\text { Nome } \\
\text { popular }\end{array} \\
\end{array}$ & $\begin{array}{c}\text { Partes } \\
\text { utilizadas }\end{array}$ & $\begin{array}{c}\text { Formas de } \\
\text { preparo }\end{array}$ & $\begin{array}{c}\text { Efeito } \\
\text { terapêutico }\end{array}$ & $\begin{array}{c}\text { Uso/ } \\
\text { Indicação } \\
\text { popular }\end{array}$ \\
\hline \multicolumn{6}{|c|}{ FABACEAE/LEGUMINOSAE } \\
\hline $\begin{array}{l}\text { Anadenanthe } \\
\text { ra colubrina } \\
\text { (Vell.) } \\
\text { Brenan. }\end{array}$ & Angico & Casca & $\begin{array}{l}\text { Decocção, } \\
\text { garrafada }\end{array}$ & $\begin{array}{l}\text { Anti- } \\
\text { inflamatório }\end{array}$ & Inflamações \\
\hline $\begin{array}{c}\text { Amburana } \\
\text { cearensis } \\
\text { (Allemão) A. } \\
\text { C. Sm. }\end{array}$ & $\begin{array}{l}\text { Cumarú, } \\
\text { imburana } \\
\text { de cheiro }\end{array}$ & $\begin{array}{l}\text { Casca do } \\
\text { caule, } \\
\text { folha, } \\
\text { semente }\end{array}$ & $\begin{array}{c}\text { Água, } \\
\text { lambedor, } \\
\text { banho, chá }\end{array}$ & $\begin{array}{l}\text { Analgésico, } \\
\text { anti- } \\
\text { inflamatório }\end{array}$ & $\begin{array}{c}\text { Dores em } \\
\text { geral, } \\
\text { inflamações } \\
\text { de garganta }\end{array}$ \\
\hline $\begin{array}{l}\text { Bauhinia } \\
\text { cheilantha } \\
\text { (Bong.) } \\
\text { Steud. }\end{array}$ & Mororó & Raiz, casca & $\begin{array}{l}\text { Decocção, } \\
\text { garrafada }\end{array}$ & $\begin{array}{c}\text { Anti- } \\
\text { inflamatório }\end{array}$ & Inflamações \\
\hline $\begin{array}{l}\text { Bowdichia } \\
\text { virgilioides } \\
\text { Kunth }\end{array}$ & Sucupira & $\begin{array}{l}\text { Casca, } \\
\text { semente }\end{array}$ & $\begin{array}{c}\text { Decocção, } \\
\text { garrafada, } \\
\text { infusão, água }\end{array}$ & $\begin{array}{c}\text { Anti- } \\
\text { inflamatório e } \\
\text { analgésico }\end{array}$ & $\begin{array}{c}\text { Inflamaccões, } \\
\text { dor de } \\
\text { garganta }\end{array}$ \\
\hline $\begin{array}{l}\text { Erythrina } \\
\text { velutina } \\
\text { Willd }\end{array}$ & Mulungu & Casca & $\begin{array}{l}\text { Decocção, } \\
\text { garrafada }\end{array}$ & $\begin{array}{c}\text { Anti- } \\
\text { inflamatório }\end{array}$ & Inflamações \\
\hline $\begin{array}{l}\text { Libidibia } \\
\text { ferrea (Mart. } \\
\text { ex Tul.) L. P. }\end{array}$ & Jucá & Casca & Decoç̧ão & $\begin{array}{c}\text { Anti- } \\
\text { inflamatório }\end{array}$ & Inflamações \\
\hline $\begin{array}{c}\text { Queiroz } \\
\text { Mimosa } \\
\text { hirsutissima }\end{array}$ & Malícia & $\begin{array}{l}\text { Planta } \\
\text { inteira }\end{array}$ & Decoç̧ão & $\begin{array}{c}\text { Anti- } \\
\text { inflamatório }\end{array}$ & $\begin{array}{c}\text { Inflamações } \\
\text { externas }\end{array}$ \\
\hline $\begin{array}{c}\text { Mart. } \\
\text { Mimosa } \\
\text { tenuiflora } \\
\text { (Will.) Poiret }\end{array}$ & $\begin{array}{l}\text { Jurema } \\
\text { preta }\end{array}$ & Casca & $\begin{array}{l}\text { Infusão, } \\
\text { decoç̧ão }\end{array}$ & $\begin{array}{c}\text { Anti- } \\
\text { inflamatório }\end{array}$ & Inflamações \\
\hline $\begin{array}{l}\text { Anadenanthe } \\
\text { ra cebil } \\
\text { (Griseb.) } \\
\text { Astschul }\end{array}$ & Angico & $\begin{array}{c}\text { Casca, } \\
\text { entrecasca }\end{array}$ & $\begin{array}{c}\text { Infusão, } \\
\text { Xarope, } \\
\text { Maceração, } \\
\text { pomada }\end{array}$ & $\begin{array}{c}\text { Anti- } \\
\text { inflamatório }\end{array}$ & Inflamações \\
\hline $\begin{array}{l}\text { Stryphnoden } \\
\text { dron } \\
\text { coriaceum } \\
\text { Benth }\end{array}$ & $\begin{array}{c}\text { Barbatimã } \\
\text { o }\end{array}$ & Casca & $\begin{array}{l}\text { Infusão, } \\
\text { xarope }\end{array}$ & $\begin{array}{c}\text { Anti- } \\
\text { inflamatório }\end{array}$ & Inflamações \\
\hline $\begin{array}{l}\text { Pithecellobiu } \\
m \\
\text { avaremotemo } \\
\text { Mart. }\end{array}$ & Babatenon & - & - & $\begin{array}{l}\text { Cicatrizante, } \\
\text { Anti- } \\
\text { inflamatório }\end{array}$ & $\begin{array}{l}\text { Sangramento } \\
\text { gengival }\end{array}$ \\
\hline $\begin{array}{c}\text { Bauhinia } \\
\text { cheilantha } \\
\text { (Bong) Steud. }\end{array}$ & Mororó & $\begin{array}{l}\text { Casca, } \\
\text { folha, } \\
\text { semente }\end{array}$ & $\begin{array}{l}\text { Infusão, } \\
\text { xarope }\end{array}$ & $\begin{array}{c}\text { Anti- } \\
\text { inflamatório }\end{array}$ & $\begin{array}{l}\text { Inflamações } \\
\text { em geral, } \\
\text { afecçỗes de } \\
\text { garganta }\end{array}$ \\
\hline $\begin{array}{l}\text { Caesalpinia } \\
\text { férrea Mart. }\end{array}$ & Jucá & $\begin{array}{l}\text { Casca, } \\
\text { fruto }\end{array}$ & $\begin{array}{c}\text { Infusão, } \\
\text { xarope, } \\
\text { maceraçẫo }\end{array}$ & $\begin{array}{c}\text { Anti- } \\
\text { inflamatório }\end{array}$ & Inflamações \\
\hline
\end{tabular}

Tabela 7. Plantas medicinais, formas de preparo, indicações terapêuticas e uso popular na odontologia, segundo os conhecimentos etnobotânico da população paraibana (LAMIACEAE a VERBENACEAE)

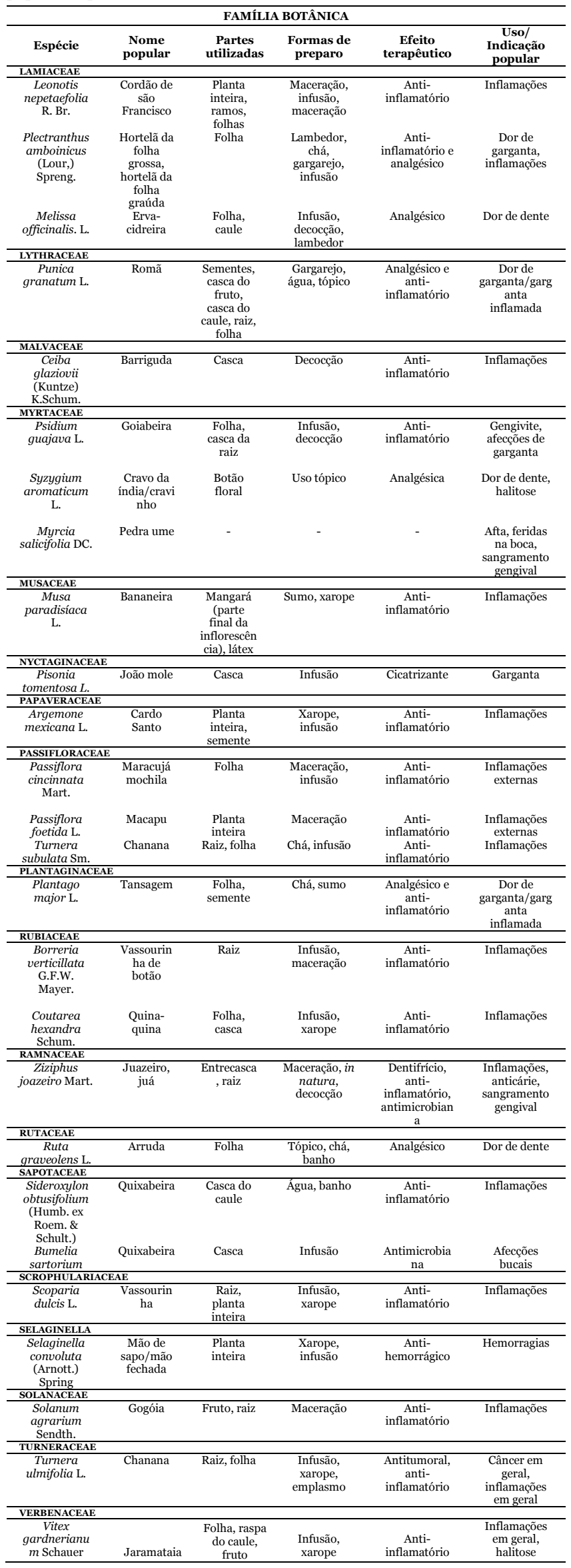

As famílias predominantes foram Fabaceae 
Bignoniaceae (5 espécies) e Anacardiaceae (4 espécies), representando aproximadamente $45 \%$ das espécies vegetais citadas. Dados apontados por Almeida Neto et al. $^{18}$, sobre plantas medicinais no Piauí, também demostraram que as famílias Fabaceae, Euphobiaceae e Anacadeaceae foram as de maior representatividade em seu estudo. Outros levantamentos etnobotânicos, realizados na região Nordeste, mostraram que pelo menos uma dessas famílias estão entre mais citadas pela população, para finalidades terapêuticas, destacando Fabaceae como a mais representativa em vários estudos ${ }^{19,20,21,22,23}$. Fabaceae e Euphobiaceae, são consideradas duas das famílias botânicas com maiores números de espécies identificados entre as angiospermas, 19.365 e 8.000 respectivamente, presentes em todos biomas, inclusive na caatinga, bioma exclusivamente brasileiro e predominante na Paraíba ${ }^{24-26}$.

Entre as recomendações terapêuticas a ação anti-inflamatória, analgésica e antimicrobiana foram as mais citadas pela população, referidas respectivamente em 46,10 e 6 espécies de plantas medicinais.

As plantas mais relatadas foram: Punica granatum L. (romã) mencionada em 7 dos 10 artigos avaliados, Anacardium occidentale L. (cajueiro roxo) presente em 6 desses artigos, e Plectranthus amboinicus Lour. Spreng. (hortelã da folha graúda) também mencionadas em 6 dos 10 artigos considerados. De acordo com Santos et al. ${ }^{16}$, essas plantas são as mais vendidas por raizeiros da capital paraibana para o tratamento de afecções odontológicas, indicadas principalmente pelo efeito anti-inflamatório e cicatrizante.

Punica granatum L. (romã), da família Punicaceae, é uma planta exótica originária do Oriente Médio e amplamente distribuída no Brasil $^{27,28}$. É conhecida por ter ação farmacológica antimicrobiana, antioxidante, antitumoral e cicatrizante, conforme demonstrado em diversos estudos $^{28-31}$. Na odontologia, é eficaz contra adesão de microrganismos cariogênicos, entre eles as bactérias Streptococcus mitis, S. mutans, S. sanguis, S. sobrinus, Lactobacillus casei e Rothia dentocariosa $^{31,32}$ e promove melhor cicatrização em úlceras bucais ${ }^{29}$.

Anacardium occidentale L. (cajueiro roxo), planta nativa do nordeste brasileiro, é facilmente encontrada em regiões semiáridas e tem importante papel econômico, cultural e medicinal para população nordestina ${ }^{33}$. Suas propriedades farmacológicas, na odontologia, são: antibacterianas ${ }^{34,35}$, antiaderente de biofilme dental ${ }^{36}$ e antifúngica ${ }^{37,38}$, com grande utilização em aftas, estomatites, inflamações e ulcerações bucais ${ }^{39}$.

Plectranthus amboinicus Lour. Spreng., popularmente denominada de hortelã da folha grossa ou hortelã da folha graúda, é uma erva pertencente à família Lamiaceae conhecida por usa ação antitussígena, antioxidante, analgésica, antimicrobiana e anti-inflamatória (referências atuais). Suas propriedades farmacológicas advêm da presença de diversas moléculas bioativas, entre elas, os monoterpenos fenólicos cravacol e timol ${ }^{40}$.

Outras espécies vegetais também demonstraram eficazes no tratamento de afecções odontológicas, entre elas Calendula officinalis L. (calêndula), Aloe vera (babosa), Plantago psyllium L. (plântago), Malva sylvestris L. (malva), Salvia officinallis (sálvia), Lippia sidoides Cham. (alecrim), Matricaria recutita L. (camomila), Enchinacea angustifólia (equinácea), Co mmiphoramolmol Engler (mirra), Mentha $x$ piperita L. (hortelã pimenta) e Ipomoea batatas (L.) Lam (batata doce) atuando no combate a dor de dente, periodontite, candidíase oral, amigdalite, aftas, estomatite, gengivite, inflamações gengival, infecções dentárias e mau hálito ${ }^{41,42}$.

O Ministério da Saúde, por meio do RENISUS (Relação Nacional de Plantas Medicinais de Interesse ao SUS), possui atualmente uma lista com 71 nomes de plantas medicinais de interesse do Sistema Único de Saúde (SUS), entre elas estão Stryphnodendron adstringens (babatenon); Anacardium occidentale L. (cajueiro); Punica granatum L. (romã), Schinus terebinthifolius (aroeira) e Allium sativum (alho) relacionadas ao tratamento de problemas odontológicos ${ }^{43}$.

As partes vegetais relatadas no preparo de remédios são: flores e inflorescência; cascas e entrecascas de caules arbóreos; cascas, sementes e látex de frutos; caules, raízes, folhas e toda estrutura vegetal; sendo as folhas e cascas de caule as mais destacadas.

O predomínio do uso de folhas para fins medicinais dá-se pelo fato de as plantas utilizadas, na maioria das vezes, são exóticas, de hábitos herbáceos, facilmente cultiváveis e com disponibilidade de folhas durante todo ano, entretanto, em espécies nativas do semiárido a preferência pelo uso da casca de caules decorre pela ausência das folhas em períodos de estiagem ${ }^{22,44}$.

Outros fatores que influenciam na escolha da estrutura vegetal, são: condições climáticas, espécie botânica a ser utilizada, praticidade na obtenção, preparo e armazenamento das partes vegetais e condições culturais da população ${ }^{22,23,44-46}$.

Quanto aos tipos de preparos medicinais o de maior destaque foi o chá, contudo outras formas também foram citadas, entre elas: xarope, garrafada, lambedor, cataplasma, pó e in natura.

O chá pode ser realizado de duas maneiras distintas, na forma de infusão, em que partes moles da planta como folhas e flores são recobertas por água quente e abafada por alguns minutos; na forma de decocção, onde estruturas rígidas da planta, 
geralmente caules e raízes, são submetidas ao processo de fervura ${ }^{47}$.

Segundo Silva et al. ${ }^{11}$, a utilização do chá como forma medicamentosa dá-se pela praticidade, facilidade e rapidez na obtenção do medicamento. Todavia, para Chaves \& Barros ${ }^{23}$, deve-se ter cautela nesta forma de preparo, uma vez que submetidas as condições de calor, princípios ativos importantes, podem ser perdidos e a eficácia do "medicamento" alterada.

A garrafada, produto medicamentoso muito vendido por raizeiros e empregado na medicina popular nordestina, é uma mistura de diferentes plantas medicinais maceradas e embebidas em água, aguardente ou vinho com finalidade de auxiliar no tratamento de problemas de saúde específicos ${ }^{48,49}$. Outro produto medicinal caseiro utilizado pela população paraibana é o cataplasma, mistura pastosa de raízes e folhas em pó adicionado em água, de uso tópico em diversos tipos de lesões e inflamações ${ }^{27}$.

Quanto aos conhecimentos acerca do uso de plantas medicinais, a literatura mostra que grande parte dos saberes são adquiridos pela livre troca de informações entre amigos, vizinhos e familiares e por meio de livros ${ }^{15,16}$. Para Rodrigues e Andrade ${ }^{19}$, a utilização de plantas medicinais de forma correta e com conhecimento prévio do médico, em patologias simples, é uma forma de aumentar a qualidade de vida, bem-estar e saúde da população e incentivar os conhecimentos etnobotânicos tradicionais.

Embora muitas espécies vegetais demonstrem atividade farmacológica eficaz no tratamento de problemas orais, ainda é escasso o número de estudos científicos acerca dos constituintes químicos e atividade biológica de plantas medicinais no nordeste brasileiro, contudo o saber popular é um meio norteador para o desenvolvimento de novas pesquisas no campo da odontologia e no tratamento de diversas doenças ${ }^{50-52}$.

\section{CONCLUSÃO}

Com base na metodologia empregada e nos resultados obtidos concluiu-se que a população paraibana detém um vasto conhecimento acerca do uso de plantas medicinais na odontologia e que estes conhecimentos constituem um caminho para ampliação de pesquisas científicas e desenvolvimento de novos fármacos.

\section{AGRADECIMENTOS}

Ao Conselho Nacional de Desenvolvimento Científico e Tecnológico (CNPq) e a Coordenação de Aperfeiçoamento de Pessoal de Nível Superior (CAPES) pelo apoio financeiro dado aos pesquisadores.

\section{REFERÊNCIAS}

1. Brasil. Ministério da Saúde. Portaria no 971, de 3 de maio de 2006. Aprova a Política Nacional de Práticas Integrativas e Complementares no
Sistema Único de Saúde. Diário Oficial da União, n. 84 , seção $1,2006.19$ p.

2. Giraldi M, Hanazaki N. Uso e conhecimento tradicional de plantas medicinais no Sertão do Ribeirão, Florianópolis, Brasil. Acta Bot Bras. 2010;24(2):395-406.

3. Martins AG, Rosário, DL, Barros MN, Jardim MAG. Levantamento etnobotânico de plantas medicinais, alimentares e tóxicas da ilha do Combu, município de Belém, estado do Pará, Brasil. Rev Bras Farm. 2005;86(1):21-30.

4. Rocha R, Marisco G. Estudos etnobotânicos em comunidades indígenas no Brasil. Rev Fitos. 2016;10(2):95-219.

5. Ministério do Meio Ambiente MMA. Caatinga. Disponível em: http://www.mma.gov.br/biomas/ caatinga. Acesso: $26 \mathrm{fev} 2018$.

6. Oliveira RCC, Silva AO, Maciel SC, Melo JRF. Situação de vida, saúde e doença da população Indígena Potiguara. Rev Min Enferm. 2012;16(1):81-90.

7. Silva JAN. Condições de moradia e de saúde em três comunidades quilombolas do estado da Paraíba. Cadernos Imbondeiro. 2015;4(1):59-70.

8. Brito MFM, Marín EA, Cruz DD. Medicinal plants in rural settlements of a protected area in the litoral of Northeastern Brazil. Ambient soc. 2017;20(1):83-104.

9. Costa JC, Marinho MGV. Etnobotânica de plantas medicinais em duas comunidades do município de Picuí, Paraíba, Brasil. Rev Bras Pl Med. 2016;18(1):125-34.

10.Souza DR, Rodrigues ECAMS. Plantas medicinais: indicações de raizeiros para tratamentos de feridas. Rev Bras Promoç Saúde. 2016;29(2):197-203.

11.Silva MDP, Marini FS, Melo RS. Levantamento de plantas medicinais cultivadas no Município de Solânea, agreste paraibano: reconhecimento e valorização do saber tradicional. Rev Bras Pl Med. 2015;14(4 Suppl 2):881-90.

12.Cordeiro JMP, Félix LP. Conhecimento botânico medicinal sobre espécies vegetais nativas da caatinga e plantas espontâneas no agreste da Paraíba - Brasil. Rev Bras Pl Med. 2014;16(3 Suppl 1):685-92.

13.Lucena CML, Lucena RFP, Costa GM, Carvalho TKN, Costa GGS, Alves RRN et al. Use and knowledge of cactaceae in Northeastern Brazil. J Ethnobiol Ethnomed. 2013;9:62.

14.Souza CMP, Brandão DO, Silva MSP, Palmeira AC, Simões MOS, Medeiros ACD. Utilização de plantas medicinais com atividade antimicrobiana por usuários do serviço público de saúde em Campina Grande - Paraíba. Rev Bras Pl Med. 2013;15(2):188-93.

15.Marinho MGV, Silva CC, Andrade LHC. Levantamento etnobotânico de plantas medicinais 
em área da caatinga no município de São José do Espinharas, Paraíba, Brasil. Rev Bras Pl Med. 2011;13(2):170-82.

16. Santos EB, Dantas GS, Santos HB, Melo Diniz MFF, Sampaio FC. Estudo etnobotânico de plantas medicinais para problemas bucais no município de João Pessoa, Brasil. Rev Bras Farmacogn. 2009;19(1b):321-24.

17.Pinheiro FA, Torres GV, Davim RMB, Xavier Filho L. Utilização das principais plantas medicinais em uma comunidade rural. $\mathrm{R}$ Bras Enferm. 1996;49(4):511-18.

18. Almeida Neto JR, Barros RFM, Silva PRR. Uso de plantas medicinais do Passa-Tempo, estado do Piauí, nordeste do Brasil. R bras Bioci. 2015; 13(3):165-75.

19. Rodrigues AP, Andrade LHC. Levantamento etnobotânico das plantas medicinais utilizadas pela comunidade de Inhamã, Pernabunco Nordeste do Brasil. Rev Bras Pl Med. 2014;16(3 suppl 1):721-30.

20.Lima IEO, Nascimento LAM, Silva MS. Comercialização de plantas medicinais do município de Arapiraca-AL. Rev Bras Pl Med. 2016;18(2):462-72.

21. Mosca VP, Loiola MIB. Uso popular de plantas medicinais no Rio Grande do Norte, Nordeste do Brasil. Rev Caatinga. 2009;22(4):225-34.

22. Ribeiro DA, Macêdo DG, Saraiva ME, Oliveira SF, Souza MMA, Menezes IRA. Potencial terapêutico e uso de plantas medicinais em uma área de caatinga no estado do Ceará, nordeste do Brasil. Rev Bras Pl Med. 2014;16(4):912-30.

23. Chaves EMF, Barros RFM. Diversidade e uso de recursos medicinais do carrasco na APA da Serra da Ibiapina, Piauí, Nordeste do Brasil. Rev Bras Pl Med. 2012;14(3):476-86.

24.Andrade ALP, Miotto STS, Santos EP. A subfamília Faboideae (Fabaceae Lindl.) no parque estadual do Guartelá, Paraná, Brasil. Hoehnea. 2009;36(4):737-68.

25.Snak C, Miotto, STS, Goldeberg R. Phaseolinae (Leguminosae, Papilionadeae, Phaseoleae) no estado do Paraná, Brasil. Rodriguésia. 2011; 62(3):695-716.

26. Sátiro LN, Roque N. A família Euphobiaceae nas caatingas arenosas do médio rio São Francisco, BA, Brasil. Acta Bot Bras. 2008;22(1):99-118.

27. Baracuhy JGV, Furtado DA, Francisco PRM, Lima JLS, Pereira JPG. Plantas medicinais de uso comum no nordeste do Brasil. $2^{\mathrm{a}}$ ed. Campina Grande: EDUFCG: 2016.

28. Menezes SMS, Pinto DM, Cordeiro LN. Atividades biológicas in vitro e in vivo de Punica granatum L. Rev Bras Med. 2008;65(11):388-91.

29. Nascimento Júnior BJ. Estudo da ação da romã (Punica granatum L.) na cicatrização de úlceras induzidas por queimaduras no dorso da língua de ratos Wistar (Rattus norvegicus). Rev Bras Pl Med. 2016;18(2):423-32.

30. Oliveira LP, Pinheiro RC, Vieira MS, Paula JR, Bara MTF, Valadares MC. Atividade citotóxica e antiangiogênica de Punica granatum L., Punicaceae. Rev Bras Farmacogn. 2010;20(2):201-7.

31.Pereira JV, Pereira MSF, Sampaio FC, Sampaio MCC, Alves PM, Araújo CRF et al. Efeito antibacteriano e antiaderente in vitro do extrato da Punica granatum linn. sobre microrganismos do biofilme dental. Rev Bras Farmacogn. 2006;16(1):88-93.

32.Ferrazzano GF, Scioscia E, Sateriale D, Pastore $\mathrm{G}$, Colicchio $\mathrm{R}$, Paglucia $\mathrm{C}$ et al. In vitro antibacterial activity of Pomegranate juice and peel extracts on cariogenic bactéria. Biomed Res Int. 2017;2017:2152749.

33. Serrano LAL, Pessoa PFAP. Aspectos econômicos da cultura do cajueiro. Sistema de Produção EMBRAPA. Disponível em: https://www.spo.cnptia.embrapa.br/conteudo?p_p _lifecycle=0\&p_p_id=conteudoportlet_WAR_sist emasdeproducaolf6_1ga1ceportlet\&p_p_col_coun $\mathrm{t}=1 \&$ p_p_col_id=column-2\&p_p_state $=$ normal \&p_r_p_-76293187_sistemaProducaoId $=7705 \&$ p_r_p_-996514994_topicoId $=10308 \& p_{-}$p $\_$mode =view>. Acesso: 21 jan 2018.

34. Silva NL, Bezerra RA, Costa FN, Rocha MMNP, Pereira SLS. Avaliação do efeito do extrato da casca do cajueiro sobre microrganismos de biofilme subgengival. Estudo experimental in vitro. Periodontia. 2013;23(4):26-30.

35. Melo AFM, Santos EJV, Souza LFC, Carvalho AAT, Pereira MSV, Higina JS. Atividade antimicrobiana in vitro do extrato de Anacardium occidentale L. sobre espécies de Streptococcus. Rev Bras Farmacogn. 2006;16(2):202-5.

36. Menezes KM, Pereira JV, Nóbrega DRM, Freitas AFR, Pereira MRV, Pereira AV. Antimicrobial and anti-adherent in vitro activity of tannins isolated from Anacardium occidentale Linn. (Cashew) on dental biolfilm bactéria. Braz Res Pediatr Dent Integr Clin. 2014;14(3):191-98.

37.Araújo CRF, Pereira MSF, Higino JS, Pereira JV, Martins AB. Atividade antifúngica in vitro da casca de Anaccardium occidentale linn. sobre leveduras do gênero cafezfendida. Arq Odontol. 2005;41(3):193-72.

38. Cardoso AMR, Cavalcanti YW, Almeida LFD, Pérez ALAL, Padilha WWN. Antifungal activity pf plant-based tintures on Candida. RSBO. 2012;9(1):25-30.

39. Araújo CRF, Pereira JV, Pereira MSV, Alves PM, Higino S, Martins AB. Concentração mínima bactericida do extrato de cajueiro sobre bactérias do biofilme dental. Pesq Bras Odontoped Clin Integr, 2009;9(2):187-91. 
40. Arumugam GA, Swamy MK, Sinniah UR. Plectranthus amboinicus (Lour.) Spreng: botanical, phytochemical, pharmacological and nutritional significance. Molecules. 2016;21(4):369.

41.Catão MHC, Silva MSP, Silva ADL, Costa RO. Estudos Clínicos com Plantas Medicinais no Tratamento de Afecções Bucais: Uma Revisão de Literatura. UNOPAR Cient Ciênc Biol Saúde 2012;14(4):279-85.

42. Gonçalves ZA, Macedo M, Lima E, Aranha AMF, Pereira ICL, Lenza JB et al. Alternativas terapêuticas para tratamento de afecções bucais no idoso. Rev Odontol Bras Central. 2014;23(66):130-34.

43. Agência Saúde. MS elabora Relação de Plantas Medicinais de Interesse ao SUS 06/03/2009. [s.l: s.n.]. Disponível em: http://bvsms.saude. gov.br/bvs/sus/pdf/marco/ms_relacao_plantas_me dicinais_sus_0603.pdf. Acesso: 02 out. 2017.

44.Franco EAP, Barros RFM. Uso e diversidade de plantas medicinais no Quilombo Olho D'água dos Pires, Esperantina, Piauí. Rev Bras Pl Med. 2006;8(3):78-88.

45.Baptistel AC, Coutinho JMCP, Lins Neto EMF, Monteiro JM. Plantas medicinais utilizadas na comunidade Santo Antônio, Currais, sul do Piauí: um enfoque etnobotânico. Rev Bras Pl Med. 2014;16(2):406-25.

46.Lima RA, Magalhães SA, Santos MRA. Levantamento etnobotânico de plantas medicinais utilizadas na cidade de Vilhena, Rondônia. Pesquisa \& Criação. 2011;10(2):165-79.

47.Brasil, Agência Nacional de Vigilância Sanitária. Formulário de Fitoterápicos da Farmacopéia Brasileira/ Agência Nacional de Vigilância Sanitária. Brasília: ANVISA, 2011. Disponível em: $\quad$ http://www.anvisa.gov.br/hotsite/ farmacopeiabrasileira/conteudo/Formulario_de_Fi toterapicos_da_Farmacopeia_Brasileira.pdf.

Acesso: $21 \mathrm{fev} 2018$.

48. Marques JO, Oliveira MFF, Lacerda GA. Efeito Alopático e análise de rótulos de garrafadas comercializados no Mercado Municipal de Montes Claros. Rev Bras Pl Med. 2015;14(4):1134-41.

49. Soares J. O gênio da garrafada. CURARECiência da Plantas Medicinais. Disponível em: https://coletivocurare.wordpress.com/2012/04/26/ o-genio-da-garrafada/. Acesso: 20 fev 2018.

50.Agra MF, et al. Medicinal and poisonous diversity of the flora of "Cariri Paraibano", Brazil. Journal of Ethnopharmacol. 2007;111(2):383-95.

51.Evangelista SS, Sampaio FC, Parente RC, Bandeira MFCL. Fitoterápicos na odontologia: estudo etnobotânico na cidade de Manaus. Rev Bras Pl Med. 2013;15(4):513-19.
52.Francisco KSF. Fitoterapia: uma opção para o tratamento odontológico. Rev Saúde. 2010;4(1):18-24.

\section{CONFLITO DE INTERESSES}

Os autores declaram não haver conflitos de interesse.

\section{AUTOR PARA CORRESPONDENCIA}

\section{Rebecca Rhuanny Tolentino Limeira}

rebecca.rhuanny@hotmail.com

Submetido em 27/09/2019

Aceito em 12/03/2019 